\title{
Deformed Oscillators with Two Double (Pairwise) Degeneracies of Energy Levels ${ }^{\star}$
}

\author{
Alexandre M. GAVRILIK and Anastasiya P. REBESH
}

Bogolyubov Institute for Theoretical Physics, 14-b Metrologichna Str., 03680 Kyiv, Ukraine

E-mail: omgavr@mail.bitp.kiev.ua

Received October 09, 2007, in final form November 13, 2007; Published online November 22, 2007

Original article is available at http://www.emis.de/journals/SIGMA/2007/112/

\begin{abstract}
A scheme is proposed which allows to obtain special $q$-oscillator models whose characteristic feature consists in possessing two differing pairs of degenerate energy levels. The method uses the model of two-parameter deformed $q, p$-oscillators and proceeds via appropriately chosen particular relation between $p$ and $q$. Different versions of quadratic relations $p=f(q)$ are utilized for the case which implies two degenerate pairs $E_{1}=E_{2}$ and $E_{3}=E_{4}$. On the other hand, using one fixed quadratic relation, we obtain $p$-oscillators with other cases of two pairs of (pairwise) degenerate energy levels.
\end{abstract}

Key words: q,p-deformed oscillators; q-oscillators; energy levels degeneracy; energy function

2000 Mathematics Subject Classification: 81S05; 81R50

\section{Introduction}

The celebrated model of harmonic oscillator in quantum mechanics is distinguished as fundamental model applicable to a plenty of real physical systems. But, when applying the harmonic oscillator for description of real systems, there is often a substantial discrepancy between theoretical results and experimental data. Therefore, to make description of a physical system more successful, it is natural to explore modifications of the model. For this goal, several deformed versions of the commutation relations of conventional harmonic oscillator have been proposed. Probably, the simplest modified version of the oscillator is the model introduced in [1], namely the Arik-Coon (AK) q-deformed oscillator. Subsequently, other types of deformed oscillators have been also written out: $q$-oscillator of Biedenharn and Macfarlane (BM) [2,3], the socalled Tamm-Dancoff (TD) deformed oscillator [4, 5], the $q, p$-oscillators [6], and more general models $[7,8,9]$.

No doubt, various deformed oscillators, both known and new ones, deserve detailed study. In our paper we analyze unusual degeneracy properties of energy levels of $q, p$-oscillators (for real $q, p)$ and obtain, on their base, certain $q$-deformed or $p$-deformed oscillators possessing the property of simultaneous two degeneracies within two certain pairs of energy levels.

A "no-go" theorem is known in quantum mechanics stating that, in one dimension, the discrete spectrum of any conventional quantum mechanical system with quadratic kinetic term and nonsingular potential $V(x)$ does not admit degeneracy of energy levels [10], see also [11] for some examples going beyond the statement. However, there certainly exist more general, than conventional, systems of quantum mechanics which possess such a property as degeneracy of some energy levels, even in the absence of any obvious symmetry (we mean so-called "accidental" degeneracy). Deformed oscillators ( $q$-oscillators, $q, p$-oscillators) can supply large class of such systems. As recently demonstrated in [12], the TD deformed oscillator is just such a special model

${ }^{\star}$ This paper is a contribution to the Proceedings of the Seventh International Conference "Symmetry in Nonlinear Mathematical Physics" (June 24-30, 2007, Kyiv, Ukraine). The full collection is available at http://www.emis.de/journals/SIGMA/symmetry2007.html 
which possesses "accidental" double degeneracy within a pair of energy levels, at appropriately fixed value of $q$. Note that the TD oscillator is contained as a reduced $q=p$ one-parameter case in the two-parameter generalized family of $q, p$-oscillators. What concerns the $q, p$-oscillator, it can be proven [13] that, for appropriate $q$ and $p$, double degeneracy within the corresponding pair of energy levels takes place. Moreover, starting from the two-parameter deformed oscillators, a plenty of $q$-deformed or $p$-deformed oscillators can be derived which manifest the property, completely analogous to the above mentioned accidental double degeneracy of the TD oscillator, occurring at definite values of $q$ or $p$.

The aim of our paper is to demonstrate the existence of special $q$-oscillator models possessing two double degeneracies (occurring within two different pairs) of energy levels, namely to describe a procedure that allows to obtain such $q$-oscillator models from the two-parameter family of $q, p$-oscillators. In most of the considered particular cases we also show graphically the shape of the energy as function of the quantum number $n$ at fixed deformation parameter(s).

The plan of the paper is the following. In Section 2 we recall necessary facts on the $q, p$ oscillator and mention the property of TD oscillators to have double degeneracy. Other particular examples of $q$-oscillators inferred using various functional dependencies $p=f(q)$, are indicated in Section 3 were different types (pairs) of degenerate energy levels are considered. In Section 4 we examine an interesting case of occurrence of two double degeneracies of particular pairs of energy levels of $q, p$-oscillators for specially fixed values of $q$ and $p$.

For each case of two double degeneracies, a graphical picture illustrating explicit dependence of energy on the quantum number $n$ is given. Last section is devoted to conclusions.

\section{Necessary facts concerning $q, p$-oscillator}

General two-parameter family of $q, p$-deformed oscillators is defined by the relations [6]

$$
\begin{aligned}
& a a^{+}-q a^{+} a=p^{N}, \quad a a^{+}-p a^{+} a=q^{N}, \\
& {[N, a]=-a, \quad\left[N, a^{+}\right]=a^{+} .}
\end{aligned}
$$

From the two relations in (1) the main formulas (invariant under $p \leftrightarrow q$ ) do follow:

$$
a^{+} a=\llbracket N \rrbracket_{q, p}, \quad a a^{+}=\llbracket N+1 \rrbracket_{q, p}, \quad \llbracket X \rrbracket_{q, p} \equiv \frac{q^{X}-p^{X}}{q-p} .
$$

We take the Hamiltonian in the form analogous to usual quantum harmonic oscillator, i.e.,

$$
H=\frac{\hbar \omega}{2}\left(a a^{+}+a^{+} a\right)
$$

From now on, $\hbar \omega=1$ is put for simplicity. The $q, p$-analogue of the Fock space, with the vacuum state $|0\rangle$ obeying $a|0\rangle=0$, is adopted. Then,

$$
|n\rangle=\frac{\left(a^{+}\right)^{n}}{\sqrt{\llbracket n \rrbracket_{q, p} !}}|0\rangle, \quad N|n\rangle=n|n\rangle
$$

where $\llbracket n \rrbracket_{q, p} !=\llbracket n \rrbracket_{q, p} \llbracket n-1 \rrbracket_{q, p} \cdots \llbracket 2 \rrbracket_{q, p} \llbracket 1 \rrbracket_{q, p}$, and the $q, p$-brackets are defined in (3). The creation and annihilation operators act by the formulas

$$
a|n\rangle=\sqrt{\llbracket n \rrbracket_{q, p}}|n-1\rangle, \quad a^{+}|n\rangle=\sqrt{\llbracket n+1 \rrbracket_{q, p}}|n+1\rangle .
$$

From (4)-(6), the spectrum $H|n\rangle=E_{n}|n\rangle$ of the Hamiltonian reads:

$$
E_{n} \equiv E_{q, p}(n)=\frac{1}{2}\left(\frac{q^{n+1}-p^{n+1}}{q-p}+\frac{q^{n}-p^{n}}{q-p}\right)=\frac{1}{2}\left(\sum_{r=0}^{n} q^{r} p^{n-r}+\sum_{s=0}^{n-1} q^{s} p^{n-1-s}\right) .
$$


At $q \rightarrow 1$ and $p \rightarrow 1$ one recovers the familiar result $E_{n}=n+\frac{1}{2}$ as it should. Moreover, at $n=0$ we have $E_{n}=\frac{1}{2}$ regardless of the values of $q$ and $p$.

For real values of $q$ and $p$ belonging to the interval $(0,1]$, we study the issue of "accidental" degeneracy of energy levels. The fact of double degeneracies of certain energy levels was first revealed in the paper [12] where we examined the degeneracies of various pairs of energy levels of TD type $q$-oscillator at the corresponding values of the parameter $q$. In [13], it is proved that $q, p$-oscillators as well exhibit double degeneracy of energy levels. For instance, it is possible to realize such degeneracy of energy levels as $E_{2}=E_{3}$, or $E_{0}=E_{4}$, or others. In that paper, we dealt with real values of $q$ and $p$ belonging to the interval $(0,1]$.

Since our present paper is focused on the study of two double degeneracies of energy levels, the easiest way to achieve this goal is to start with two-parameter $q, p$-oscillators and then reduce them in a definite way to appropriate one-parameter $q$-oscillator or $p$-oscillator.

\section{Two double (pairwise) degeneracies of energy levels}

By exploiting the $q$-dependence of energy spectrum of various $q$-deformed oscillators we can find that simultaneous degeneracy of more then one pair of energy levels is possible. In this section, we develop a procedure for obtaining those special $q$ - (or $p$-) oscillators which possess the unusual property of two double degeneracies (within each of two pairs) of energy levels.

So, let us take the $q, p$-oscillators defined in (1)-(3) as our playground. In our whole treatment, $q$ and $p$ attain real values from the interval $(0,1]$. Obviously, the limit $q \rightarrow 1, p \rightarrow 1$ recovers usual quantum oscillator.

It can be proved that the relation $E_{n}-E_{n+k}=0$, with $n$ and $k$ being some fixed non-negative integers, determines a continuous curve in the $(q, p)$-plane. The set of all such curves is naturally divided into two sets (families):

$$
\text { 1) } n \neq 0, \quad k \geq 1, \quad \text { 2) } n=0, \quad k \geq 2 .
$$

The curves from the first family do not intersect with each other except for the two points $(1,0)$ and $(0,1)$ which are nothing but the endpoints for each curve. The curves from the second family have no common points; as $k$ grows from 2 to infinity, their endpoints move along the both axes, starting from the value $(\sqrt{5}-1) / 2$ at $k=2$ and approaching at asymptotically large $k$ to 1 , on each axis. Both the first and the second families in (8) share the property that the sequence of curves which correspond to growing integer indices $n+k$ and $k$ respectively, have the tendency of squeezing into the upper right corner given by the vertex $(1,1)$ and formed by the straight lines $q=1, p=1$.

Now we go over to main point of our scheme: we shall apply different appropriate choices of functional dependence $p=f(q)$. This way, from general two-parameter $q, p$-oscillator we can obtain various versions of one-parameter oscillator. The particular case studied in [12] is nothing but the $p=q$ reduction (TD oscillator), and in this paper we exploit other special dependencies $p=f(q)$ which can lead to the desired degeneracy properties. Besides, we often visualize the explicit energy spectrum, with the occurring degeneracies, of those one-parameter oscillators that are obtained by exploring a particular choice of $p=f(q)$.

\section{$3.1 q$-oscillator resulting from quadratic (parabolic) relation $p=f(q)$}

(i) Let us take

$$
p=\alpha(q-\beta)^{2}+\gamma,
$$

where $\alpha, \beta, \gamma$ are the parameters of the curve. By inserting this in basic formulas for $q, p$ oscillators, a particular one-parameter set of new deformed oscillators is obtained. The resulting 
Table 1. Values of the parameters in equation (12) corresponding to $p=p_{0}$.

\begin{tabular}{|c|c|c|c|c|c|}
\hline & $q_{1}$ & $q_{2}$ & $\alpha$ & $\beta$ & $\gamma$ \\
\hline$p_{0}=0.6$ & 0.554400 & 0.900317 & 9.005207 & 0.727359 & 0.330613 \\
\hline
\end{tabular}

$q^{-}$(or p-) oscillators will possess the property of two double degeneracies of energy levels, if the simultaneous degeneracy within each of, e.g., the following two pairs does occur:

$$
E_{1}=E_{2}, \quad E_{3}=E_{4}
$$

(note that any other concrete two pairs of energy levels, except for $E_{0}=E_{1}$, can be chosen).

In order that such two-fold double degeneracy (10) of energies would emerge, the curve corresponding to relation (9) whose effect consists in reducing the $q, p$-oscillator to some oneparameter $q$ - or $p$-oscillator, should cross the curves $E_{2}-E_{1}=0$ and $E_{4}-E_{3}=0$ at the points $\mathrm{A}\left(q_{1}, p_{1}\right)$ and $\mathrm{B}\left(q_{2}, p_{2}\right)$ respectively, where $p_{1}=p_{2}=p_{0}$. Here, the coordinates $q_{1}$ and $q_{2}$ are evaluated, at fixed $p_{0}$, from solving the equations (10). Moreover, the curve (9) should contain the point $(1,1)$ in order that $p(1)=1$ since, obviously, each $q$ - or $p$ - oscillator at $q \rightarrow 1$ and $p \rightarrow 1$ must turn into the usual harmonic oscillator.

Then, the following system of equations (recall, $p_{1}=p_{2}=p_{0}$ )

$$
\alpha\left(q_{1}-\beta\right)^{2}+\gamma=p_{1}, \quad \alpha\left(q_{2}-\beta\right)^{2}+\gamma=p_{2}, \quad \alpha(1-\beta)^{2}+\gamma=1,
$$

is used for finding $\alpha, \beta, \gamma$ that specify the curve in (9). The solution of the system reads:

$$
\alpha=\frac{1-p_{0}}{\left(1-q_{1}\right)\left(1-q_{2}\right)}, \quad \beta=\frac{q_{1}+q_{2}}{2}, \quad \gamma=1+\frac{\left(p_{0}-1\right)}{\left(1-q_{1}\right)\left(1-q_{2}\right)}\left(1-\frac{q_{1}-q_{2}}{2}\right)^{2} .
$$

We put these $\alpha, \beta$ and $\gamma$ in the parabolic relation (9) and get it completely specified. Then, by inserting the inverted relation $q=f^{-1}(p)$ into the defining formulas (1)-(3) of $q, p$-oscillator we derive a special $p$-oscillator such that at $p=p_{0}$ the desired two-fold double degeneracy (i.e., the simultaneous degeneracies (10) of two different pairs of energy levels) is guaranteed.

To illustrate the obtained pattern of degeneracies let us fix for example $p=p_{0}=0.6$. Put this value of $p_{0}$ into equations in (10) and solve them for $q_{1}$ and $q_{2}$. As result, the numerical values of $q_{1}$ and $q_{2}$ are at our disposal (we give them up to 6 digits). With these $p_{0}, q_{1}$ and $q_{2}$ inserted in (12) we find $\alpha, \beta$ and $\gamma$; the entire set of values is placed in Table 1.

In Fig. 1 (left) the resulting picture of two-fold double degeneracy, see (10), is shown.

Note that the admissible values of $p$ for the $p$-oscillator are such that $p \in\left[p_{\min }, 1\right]$ where $p_{\text {min }}$ is the minimal value of the function (9) whose image is the parabola. In case at hands,

$$
p_{\min }=\gamma=0.330613 \text {. }
$$

It is of interest to visualize the behavior of energy $E_{n}=E(n)$ as function of $n$ for the so obtained $p$-oscillator. Recall that the specified values $q_{1}, q_{2}, \alpha, \beta, \gamma$ are determined at the fixed $p_{0}$. Since a one-parameter deformed oscillator possesses, by construction, the desired property of two double degeneracies occurring at the same $p=p_{0}$, we should end up (not with the $q_{-}^{-}$, but) with the $p$-oscillator. Therefore, we invert the considered parabolic relation: taking $q=f^{-1}(p)$, we substitute this in equation (7). Since the inverse $f^{-1}(p)$, given as

$$
q= \pm \sqrt{\frac{p-\gamma}{\alpha}}+\beta
$$

contains the " \pm " type ambiguity through pre-factor of square root (accordingly, we have right or left branch of the parabola in Fig. 1 (left)), one should be careful when inserting this $f^{-1}(p)$ 

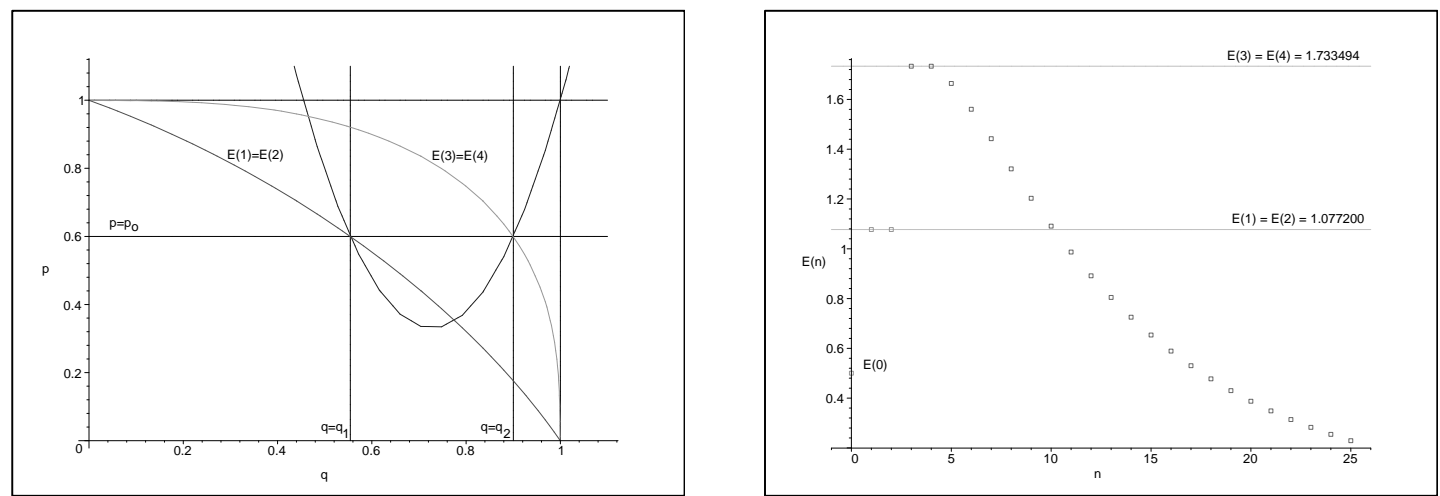

Figure 1. Left: Two double degeneracies of energy levels $E_{1}=E_{2}$ and $E_{3}=E_{4}$ occurring at the fixed $p \equiv p_{0}=0.6$. The parabolic curve $p=f(q)$ is given by (9) with $\alpha, \beta$ and $\gamma$ taken from Table $1\left(q_{1}, q_{2}\right.$ are there, too). The parabola goes through the points $\left(q_{1}, 0.6\right),\left(q_{2}, 0.6\right)$ and $(1,1)$ as it should. Right: Energy spectrum $E_{p}(n)$, at $p=0.6$, given by the expression with "+" or "-" in (13) respectively for $n=0,1,2$ or $n=3,4,5, \ldots$ (see main text). The two degeneracies $E_{1}=E_{2}$ and $E_{3}=E_{4}$ are evident.

Table 2. Values of the parameters in equation (14) corresponding to $p=p_{0}$.

\begin{tabular}{|c|c|c|c|c|c|}
\hline & $q_{1}$ & $q_{2}$ & $\tilde{a}$ & $\tilde{b}$ & $\tilde{c}$ \\
\hline$p_{0}=0.6$ & 0.554400 & 0.900317 & -0.755814 & 28.020856 & 0.727359 \\
\hline
\end{tabular}

into $E_{q, p}(n)$ in (7): the two signs result in two expressions for the one-parameter $E_{p}(n)$. Namely, the "minus" square root enters the $E_{p}(n)$ for $n=0,1,2$, whereas the "plus" sign determines $E_{p}(n)$ for the rest of levels $n=3,4,5, \ldots$. The resulting energy spectrum $E_{p}(n)$, at $p=0.6$, with the occurring two degeneracies $E_{1}=E_{2}$ and $E_{3}=E_{4}$ is shown in Fig. 1 (right).

\subsection{Hyperbolic/elliptic relations $p=f(q)$ and the $p$-oscillators possessing two double degeneracies}

For completeness and also for comparison it is worth to consider, besides parabolic, the other two cases of quadratic dependence of $p$ on $q$ : the hyperbolic and the elliptic ones.

(ii) Let us take

$$
(p-\tilde{a})^{2}-\tilde{b}(q-\tilde{c})^{2}=R^{2} .
$$

For convenience, let $R=1$ for what follows. Again we are able to design a $p$-oscillator possessing two simultaneous double degeneracies. Fixing $p$ in equations (10) as, say, $p_{0}=0.6$ we solve them to get $q_{1}=0.554400, q_{2}=0.900317$. Then we compose relevant system of three equations analogous to (11), but now encoding the fact that the hyperbolic curve runs through the points $\mathrm{A}\left(q_{1}, p_{1}\right), \mathrm{B}\left(q_{2}, p_{2}\right), \mathrm{C}(1,1)$, where $p_{1}=p_{2}=p_{0}$. Solving this system, we find the parameters $\tilde{a}$, $\tilde{b}, \tilde{c}$ in (14). The resulting set of numerical values is collected in Table 2.

With the obtained data we arrive at the picture shown in Fig. 2 (left). There, the two-fold double degeneracy is manifest (now got due to hyperbolic relation $p=f(q)$ ) it is the two branches of hyperbola that again do the job). For clarity, the value $p_{0}=0.6$ is also indicated.

The minimal admissible positive value $p_{\text {min }}$ for the $p$-oscillator obtained by inserting $q=$ $f^{-1}(p)$ of this hyperbolic relation is found from

$$
\left(p_{\min }-\tilde{a}\right)^{2}-\tilde{b}(Q-\tilde{c})^{2}=1^{2},
$$

where $Q=\frac{q_{1}+q_{2}}{2}$; for $q_{1}, q_{2}$ and $\tilde{a}, \tilde{b}, \tilde{c}$ see Table 2 . With these values, $p_{\min }=0.244186$. 
Table 3. Values of the parameters in equation (15) corresponding to $p=p_{0}$ and $\varepsilon=0.1$.

\begin{tabular}{|c|c|c|c|c|c|}
\hline & $q_{1}$ & $q_{2}$ & $\mu$ & $\nu$ & $\rho$ \\
\hline$p_{0}=0.6$ & 0.554400 & 0.900317 & 0.727359 & 1.355234 & 0.294877 \\
\hline
\end{tabular}
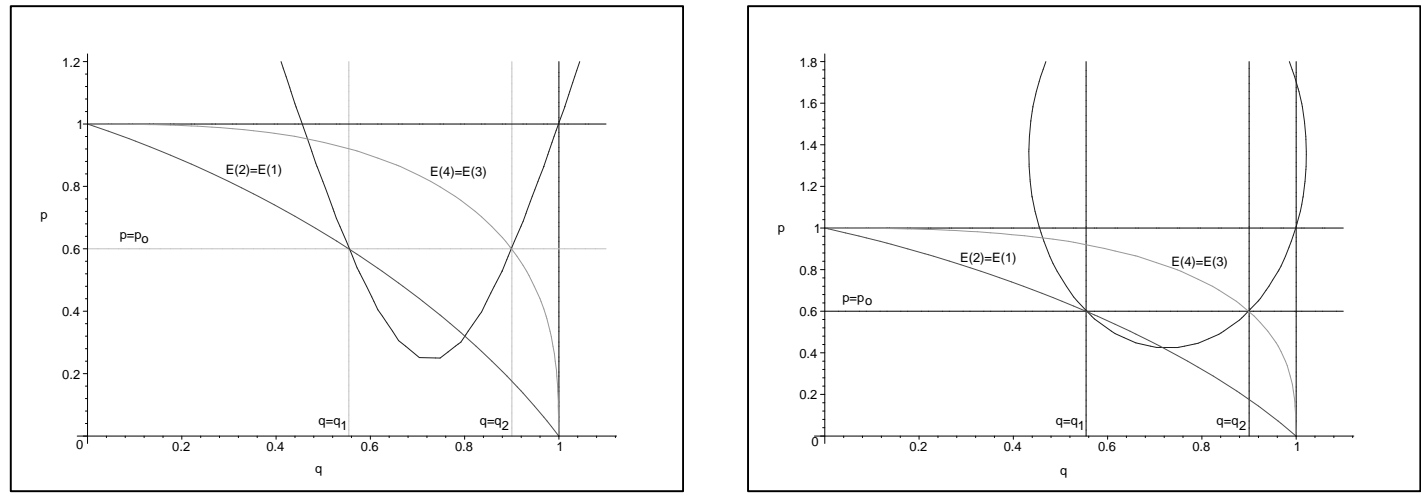

Figure 2. Left: Same as on Fig. 1 (left), but now using hyperbolic relation (14) with $\tilde{a}, \tilde{b}$ and $\tilde{c}$ given in Table 2. As seen, the curve goes through the points $\left(q_{1}, 0.6\right),\left(q_{2}, 0.6\right)$ and $(1,1)$. Right: Same as on the left panel, but here the elliptic relation (15) is used, with $\varepsilon=0.1$ and $\mu, \nu, \rho$ given in Table 3 .

The points located below $p=p_{\text {min }}$ can't belong to the chosen curve and thus are not admitted for the obtained $p$-oscillator; for those points $(q, p)$ for which $p<p_{\min }$, we have to construct another one-parameter oscillator, using the relation $p=f(q)$ compatible with fixed $p_{0}$ and, then, with the corresponding values $q_{1}, q_{2}, \tilde{a}, \tilde{b}, \tilde{c}$.

(iii) Now let us take

$$
(q-\mu)^{2}+\varepsilon(p-\nu)^{2}=\rho^{2},
$$

where $\mu, \nu, \rho, \varepsilon(\varepsilon>0$ for this elliptic case) are the relevant parameters. By solving the system of equations composed analogously to (11) these parameters are easily found and for $p_{0}=0.6$ placed in Table 3.

The present case based on the elliptic relation $p=f(q)$ in (15) is illustrated in Fig. 2 (right).

Our goal in this section was to construct special one-parameter deformed oscillators which possess the property of two-fold double degeneracy of some two pairs of energy levels. Here, the two pairs $E_{1}=E_{2}$ and $E_{3}=E_{4}$ where chosen, and we have succeeded to realize the goal. Namely, starting from the two-parameter $q, p$-oscillators defined in (1)-(3), by imposing different types of the quadratic relations $p=f(q)$ : parabolic, hyperbolic and elliptic, we have inferred the corresponding versions of one-parameter $p$-deformed oscillators, all of them sharing the property that simultaneously $E_{1}=E_{2}$ and $E_{3}=E_{4}$.

\section{$4 p$-oscillators with other pairs of degenerate energy levels}

The pairs $E_{1}=E_{2}$ and $E_{3}=E_{4}$ considered in the previous section, serve as typical examples belonging to the first family in (8). In this section, we derive and explore one-parameter deformed oscillators with similar property of two-fold double degeneracy, but for another two pairs of energy levels being specified. Moreover, we will distinguish the following two cases: (a) the both pairs of energy levels belong to the second family in (8); (b) the two pairs are taken from different families (i.e., mixed case). 
Table 4. Values of the parameters in equation (9) corresponding to $p=p_{0}$ and equation (16).

\begin{tabular}{|c|c|c|c|c|c|}
\hline & $q_{1}$ & $q_{2}$ & $\alpha$ & $\beta$ & $\gamma$ \\
\hline$p_{0}=0.4$ & 0.264365 & 0.721012 & 2.923499 & 0.492688 & 0.247594 \\
\hline
\end{tabular}
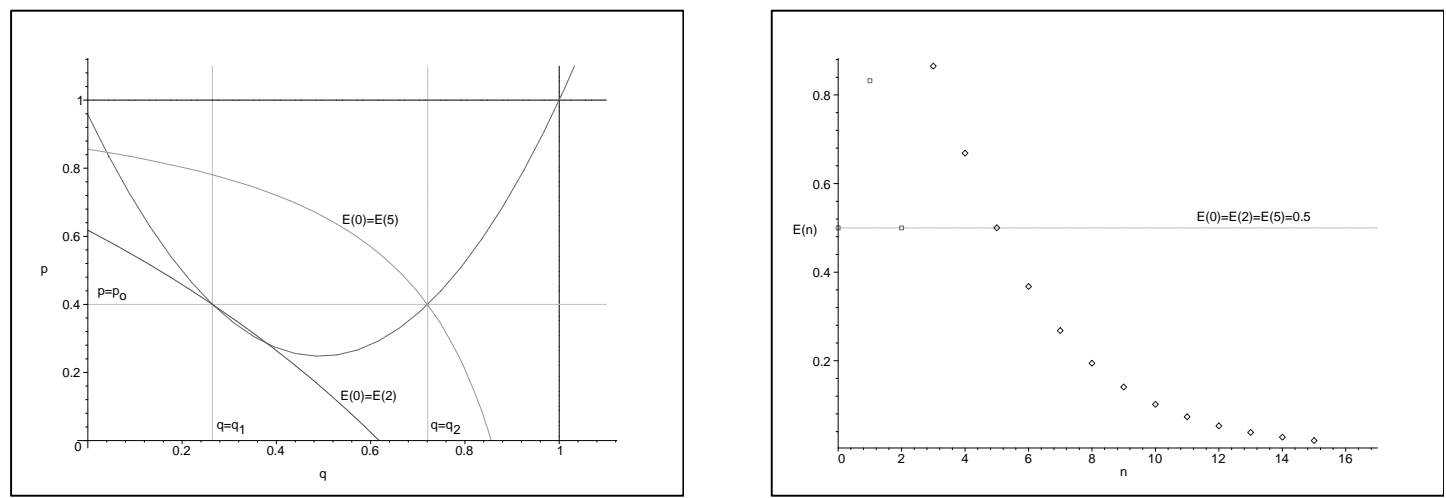

Figure 3. Left: Intersection, at $p_{0}=0.4$, of the curves $E_{2}-E_{0}=0$ and $E_{5}-E_{0}=0$ by the parabolic curve (9) whose parameters $\alpha, \beta$ and $\gamma$ are given in Table 4. Right: Simultaneous degeneracies, at $p=0.4$, of the levels $E_{2}=E_{0}$ and $E_{5}=E_{0}$ in the energy spectrum of the derived $p$-oscillator. Two formulas for $E_{p}(n)$, one for $n=0,1,2$, the other for $n=3,4,5, \ldots$, realize the " \pm " dichotomy in (13).

\section{$4.1 p$-oscillators from relation $p=f(q)$ : other pairs of energy levels}

Here we construct, using only the parabolic relation (9) of $p$ and $q$, the $p$-deformed oscillator possessing two double degeneracies for the cases in which the both pairs of energy levels belong to the first family in (8).

That is, following the same procedure as above, let us fix some two pairs (each one degenerate, as we require) of energy levels from the second family in (8), say,

$$
E_{2}-E_{0}=0, \quad E_{5}-E_{0}=0 .
$$

With some fixed $p=p_{0}$, let it be $p_{0}=0.4$, we solve the equations in (16) for $q_{1}, q_{2}$ and get: $q_{1}=0.264365, q_{2}=0.721012$. Taking these into account, from the formulas (12) we find $\alpha, \beta$ and $\gamma$ which specify the parabolic curve (9), see Table 4 for the relevant set of data.

The resulting $p$-deformed oscillator does possess the prescribed pattern of degeneracies (16), and in Fig. 3 (left) this is neatly seen. The shape of the related energy spectrum $E_{p}(n)$ is presented in Fig. (3) (right). Note the peculiar feature of non-monotonicity of the energy function which is caused by the fact that, because of the " \pm " pre-factor of square root in (13), one should use the two alternative expressions for $E_{p}(n)$ when $n=0,1,2$ or when $n=3,4,5, \ldots$

Let us finally explore the mixed case. We use again the parabolic relation (9) of $p$ and $q$, and construct the $p$-deformed oscillator which possesses the two-fold double degeneracy for the case when the two pairs belong to different families in (8). As a typical example let us take

$$
E_{3}-E_{2}=0, \quad E_{4}-E_{0}=0 .
$$

Following the same procedure as above, having fixed $p=p_{0}$ as, e.g., $p_{0}=0.4$, we solve the equations in (17) and obtain: $q_{1}=0.640778, q_{2}=0.916515$. Taking these into account, from the formulas (12) we find $\alpha, \beta, \gamma$ (see Table 5), which completely specify the parabolic curve (9) in this case.

As should, the resulting $p$-deformed oscillator acquires the prescribed pattern (17) of degeneracies. In Fig. 4 (left) this pattern is clearly seen. The shape of the related energy spectrum $E_{p}(n)$ is shown in Fig. 4 (right). Note again the peculiar feature of non-monotonicity of 
Table 5. Values of the parameters in equation (9) corresponding to $p=p_{0}$ and equation (17).

\begin{tabular}{|c|c|c|c|c|c|}
\hline & $q_{1}$ & $q_{2}$ & $\alpha$ & $\beta$ & $\gamma$ \\
\hline$p_{0}=0.4$ & 0.640778 & 0.916515 & 20.006946 & 0.778648 & 0.019714 \\
\hline
\end{tabular}
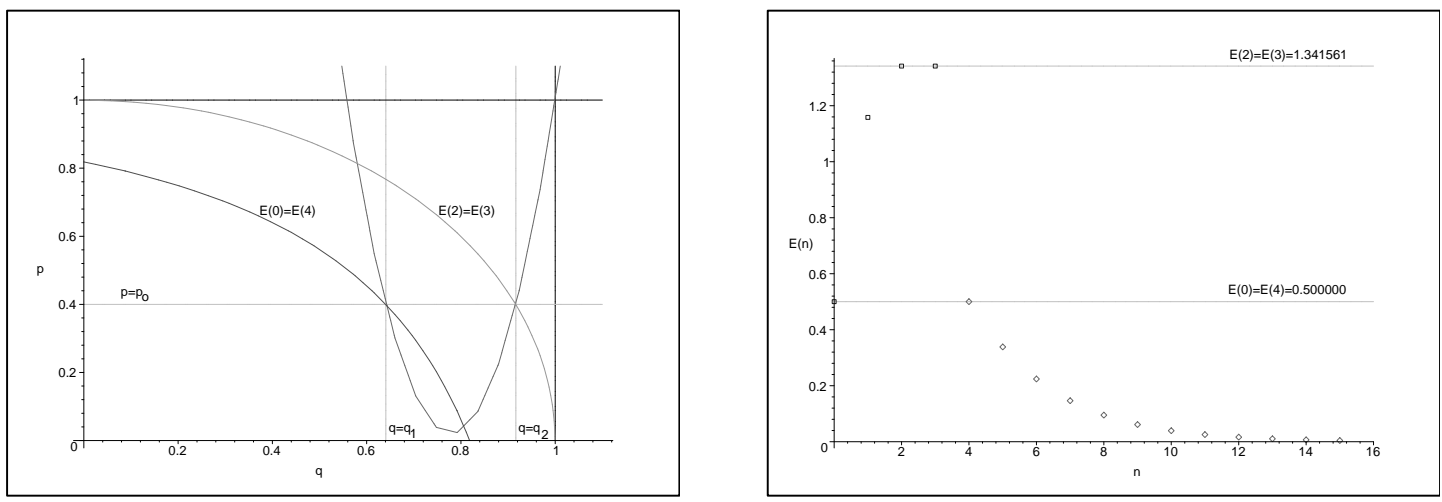

Figure 4. Left: Intersection of the curves $E_{4}-E_{0}=0$ and $E_{3}-E_{2}=0$ by the parabolic curve (9), at fixed $p=p_{0}=0.4$ and with $q_{1}, q_{2}, \alpha, \beta$ and $\gamma$ given in Table 5. Right: Energy spectrum at $p=0.4$, manifesting simultaneous degeneracies of the two pairs of energy levels $E_{4}=E_{0}$ and $E_{3}=E_{2}$.

the energy function caused by the fact that, because of the " \pm " pre-factor of square root in (13), one should use the proper version of the two alternatives for $E_{p}(n)$ : one for $n=0,1,2,3$, the other for $n=4,5, \ldots$

\section{$4.2 \quad q$-oscillator with two double degeneracies from linear relation of $p$ and $q$}

Above, we constructed one-parameter oscillators on the base of the two-parameter $q, p$-oscillator by imposing specified quadratic relation $p=f(q)$. It turns out however that two-fold double degeneracy can be gained at the two-parameter level (q,p-deformed level) more simply, for two definite (not arbitrary) pairs of energy levels. This is due to the fact that certain two curves belonging to different families of $E_{n+k}-E_{n}=0$ in (8) may merely intersect each other. Indeed, this occurs if one curve is given by $E_{k}-E_{0}=0$ from the second family in (8) with $k$ being high enough, and the other curve taken from the set $E_{n+l}-E_{n}=0, n \neq 0$, with an appropriate rather small $n$ and $l$.

In such cases it may occur that the specified curves have simultaneously two different (mutually $p \leftrightarrow q$ symmetric) points of intersection. This implies the following: simultaneous degeneracies within two certain pairs of energy levels takes place at two distinct couples of fixed $q, p$ values.

The coordinates of the points where the curves $E_{10}-E_{0}=0$ and $E_{3}-E_{1}=0$ mutually intersect are found from the equations

$$
E_{10}=E_{0}, \quad E_{3}=E_{1} .
$$

For the two intersection points (A) and (B) this yields:

$$
\begin{array}{ll}
\text { (A) } q_{1}=0.567239, \quad p_{1}=0.823554 ; & \text { (B) } q_{2}=0.823554, \quad p_{2}=0.567239 .
\end{array}
$$

Therefore, for either the pair (A) or the pair (B) in (19), the $q, p$-oscillator shows two double degeneracies from (18). For more clearness we illustrate this fact graphically in Fig. 5 (left). Besides, for obtaining one-parameter $q$-oscillator, it is now enough to take the relation $p=f(q)$ as linear function: $p=\alpha q+\beta$, with $\alpha$ and $\beta$ being such that the straight line passes through the 

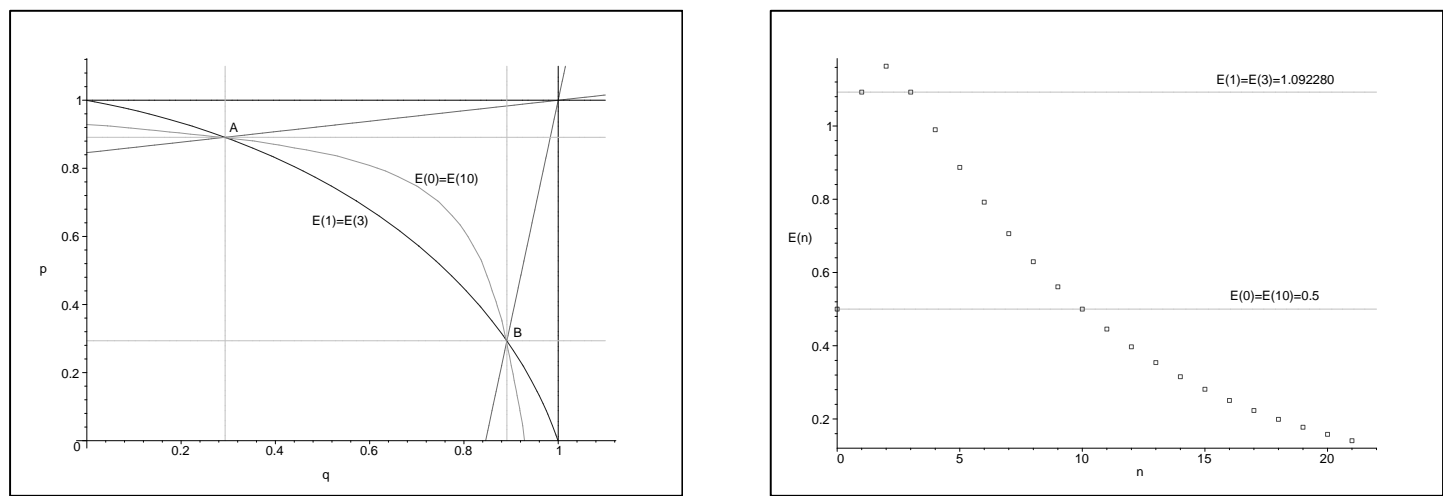

Figure 5. Left: Intersection of the curves $E_{10}-E_{0}=0$ and $E_{3}-E_{1}=0$ at the points $\mathrm{A}\left(q_{1}, p_{1}\right)$ and $\mathrm{B}\left(q_{2}, p_{2}\right)$, see (19) for numerical values, provides simultaneous degeneracies within the respective pairs of energy levels. Right: The shape of energy spectrum $E_{n}=E_{q, p}(n)$ of $q, p$-oscillator with $q_{1}, p_{1}$ or $q_{2}, p_{2}$ taken from (19). As seen, $E_{0}=E_{10}$ and $E_{1}=E_{3}$.

point $(1,1)$ and any one of the intersection points (A or B) of the considered curves, see Fig. 5 (left). The parameters $\alpha$ and $\beta$ satisfy one of the two systems of equations

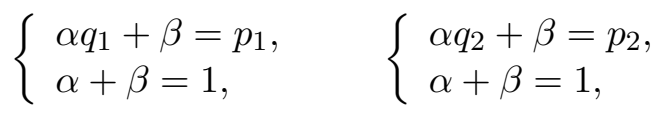

so that the result is, respectively,

$$
(\mathrm{A}) \leftrightarrow \alpha=0.407722, \beta=0.592278 ; \quad(\mathrm{B}) \leftrightarrow \alpha=2.452649, \beta=-1.452649 .
$$

Substitution of the values from (19), either the first pair or the second, into the formula (7) for the energy $E_{n}=E_{q, p}(n)$ yields the actual shape of energy function, as shown in Fig. 5 (right). Note the monotonicity of energy function of one-parameter deformed oscillator got from the linear relation. If we exploit the relation $p=\alpha q+\beta$ with $\alpha$ and $\beta$ of the case (A) in (20), i.e. the line passing through $(1,1)$ and the point $(\mathrm{A})$, we get the $q$-oscillator by excluding the parameter $p$, or get the $p$-oscillator by excluding $q$ where $q=\alpha^{-1}(p-\beta)$. Though being on equal footing, the two versions of one-parameter oscillator differ in the range of possible values of $q$ versus those of $p$ : indeed, $q \in(0,1]$ for the $q$-oscillator whereas $p \in\left[p_{\min }, 1\right], p_{\min }=\beta$, for the $p$-oscillator. The situation which arises if instead of (A) one uses the case (B) in (20), is completely analogous to the previous case of (A): this follows from the symmetry $q \leftrightarrow p$.

\section{Conclusions}

By explicit treatment, we have confirmed that peculiarities of the energy spectrum of $q$-deformed oscillator crucially depend on both the particular version of $q$-oscillator and, within each version, on the specified value of deformation parameter. As it was shown in [12], the special TD (TammDancoff) version of $q$-deformed oscillator admits the possibility of appearance, for proper $q$, of the "accidental" double degeneracy within any one chosen pair of energy levels. In the present paper, we have demonstrated how to obtain one-parameter $p$-deformed oscillators with the property of simultaneous degeneracy within each of the two specified pairs of energy levels. Having chosen two pairs of energy levels, in particular, those given in (10), we applied different cases of quadratic relation $p=f(q)$ : parabolic, hyperbolic and elliptic ones, to the two-parametric $q, p$ oscillator. On the other hand, with the specified parabolic dependence we treated and obtained the cases of $p$-oscillator such that the pairs of energy levels (16) or (17) exhibit the two-fold double degeneracy. 
We have also found that two double degeneracies can be achieved in the general two-parameter case, without imposing any particular relation $p=f(q)$, but only if the two pairs of energy levels belong to some more special subset. The typical example, $E_{0}=E_{10}$ and $E_{1}=E_{3}$ in (18), has been considered and shown to provide the intersection points $\mathrm{A}\left(q_{1}, p_{1}\right)$ and $\mathrm{B}\left(q_{2}, p_{2}\right)$ in the $(q, p)$ plane which lead, in the two-parameter case, to the desired two double (pairwise) degeneracies. These same two degeneracies $E_{0}=E_{10}$ and $E_{1}=E_{3}$ do hold for some one-parameter reduction in which the $q$-oscillator is drawn by imposing linear relation $p=\alpha q+\beta$.

The obtained energy spectra are mainly given by non-monotonic function: as seen from Fig. 1 (right), Fig. 3 (right), Fig. 4 (right), in each of these cases the energy function $E_{p}=E_{p}(n)$ consists of two distinct parts, and this is rooted in the " \pm " ambiguity of the inversion $q=f^{-1}(p)$, see (13) for example. In contrast, the situation described in Section 4.2 results in the monotonic energy function $E_{p}(n)$ since there is no ambiguity in inverting the linear dependence: $q=f^{-1}(p)$.

It is worth to point out that in our paper we have in fact also encountered the case of threefold energy level degeneracy, see Fig. 3 (right), where the energies $E_{0}, E_{2}$ and $E_{5}$ do coincide. Clearly, the proposed approach to the deformed $q, p$-oscillators and their various one-parameter reductions is suitable for exploration of the degeneracies of energy levels occurring in more complicated patterns such as, e.g., three double (pairwise) degeneracies and others.

Let us make a remark concerning the "no-go" theorem mentioned in the Introduction. The fact that $q, p$-oscillators, their special $p=q$ case known as the TD oscillator, and the new deformed oscillator models treated in this paper, really circumvent the theorem and exhibit various patterns of "accidental" degeneracies, is well explained by more involved structure of those quantum-mechanical systems where relevant features should appear: position dependent mass, the potential being a function of both coordinate and momentum, etc. (see e.g., [7, 14, 15]).

It would be of interest to analyze possible peculiarities of applying, along the lines similar to those in $[16,17,18]$, the respective versions of $q$-Bose gas model based on the above constructed novel, nonstandard $q$-oscillators, in order to examine their efficiency for description of the nonBose type features shown by the data on pion correlations collected in experiments on relativistic heavy ion collisions. Certainly, other applications of the exotic deformed oscillators treated in this paper are worth of detailed study.

\section{Acknowledgements}

This work was done under partial support of the Grant Number 14.01/016 of the State Foundation of Fundamental Research of Ukraine.

\section{References}

[1] Arik M., Coon D.D., Hilbert spaces of analytic functions and generalized coherent states, J. Math. Phys. 17 (1976), 524-527.

[2] Biedenharn L.C., The quantum group $S U_{q}(2)$ and a $q$-analogue of the boson operators, J. Phys. A: Math. Gen. 22 (1989), L873-878.

[3] Macfarlane A.J., On $q$-analogues of the quantum harmonic oscillator and the quantum group $S U_{q}(2)$, J. Phys. A: Math. Gen. 22 (1989), 4581-4585.

[4] Odaka K., Kishi T., Kamefuchi S., On quantization of simple harmonic oscillator, J. Phys. A: Math. Gen. 24 (1991), L591-L596.

[5] Chaturvedi S., Srinivasan V., Jagannathan R., Tamm-Dancoff deformation of bosonic oscillator algebras, Modern Phys. Lett. A 8 (1993), 3727-3734.

[6] Chakrabarti A., Jagannathan R., A q,p-oscillator realization of two-parameter quantum algebras, J. Phys. A: Math. Gen. 24 (1991), L711-L718.

[7] Mizrahi S.S., Camargo Lima J.P., Dodonov V.V., Energy spectrum, potential and inertia functions of a generalized f-oscillator, J. Phys. A: Math. Gen. 37 (2004), 3707-3724. 
[8] Man'ko V.I., Marmo G., Sudarshan E.C.G., Zaccaria F., f-oscillators and nonlinear coherent states, Phys. Scripta 55 (1997), 528-541, quant-ph/9612006.

[9] Meljanac S., Milekovic M., Pallua S., Unified view of deformed single-mode oscillator algebras, Phys. Lett. B 328 (1994), 55-59, hep-th/9404039.

[10] Landau L.D., Lifshitz E.M., Quantum mechanics (nonrelativistic theory), Fiz.-Mat. Lit., Moscow, 1963 (in Russian).

[11] Kar S., Parwani R.R., Can degenerate bounds states occur in one dimensional quantum mechanics?, Europhys. Lett. 80 (2007), no. 3, 30004, 5 pages, arXiv:0706.1135.

[12] Gavrilik A.M., Rebesh A.P., A q-oscillator with "accidental" degeneracy of energy levels, Modern Phys. Lett. A 22 (2007), 949-960, quant-ph/0612122.

[13] Gavrilik A.M., Rebesh A.P., Occurrence of pairwise energy level degeneracies in q,p-oscillator model, submitted.

[14] Quesne C., Tkachuk V.M., Deformed algebras, position-dependent effective masses and curved spaces: an exactly solvable Coulomb problem, J. Phys. A: Math. Gen. 37 (2004), 4267-4281, math-ph/0403047.

[15] Lorek A., Wess J., Dynamical symmetries in q-deformed quantum mechanics, Z. Phys. C67 (1995), 671-680, q-alg/9502007.

[16] Gavrilik A.M., Combined analysis of two- and three-particle correlations in the $q, p$-Bose gas model, SIGMA 2 (2006), 074, 12 pages, hep-ph/0512357.

[17] Anchishkin D.V., Gavrilik A.M., Iorgov N.Z., Two-particle correlations from the q-boson viewpoint, Eur. J. Phys. C. 7 (2000), 229-238, nucl-th/9906034.

[18] Anchishkin D.V., Gavrilik A.M., Iorgov N.Z., q-boson approach to multiparticle correlations, Modern Phys. Lett. A 15 (2000), 1637-1646, hep-ph/0010019. 